\title{
Practical Aspects on Use of Sharesource in Remote Patient Management
}

\author{
Valérie Jotterand Drepper \\ Division of Nephrology, Geneva University Hospital, Geneva, Switzerland
}

\begin{abstract}
Remote patient management (RPM) has been increasingly implemented in the care of chronic patients in the last decades with significant positive impact on clinical outcomes. Homebased dialysis therapies constitute an attractive alternative to in-center hemodialysis as they offer patients more flexibility and empowerment. Nonetheless, their remote nature and intrinsic problematic visibility to therapy-related issues occurring at home contribute to holding back both patients and nephrologists from adopting them. RPM has recently become available in automated peritoneal dialysis (APD) by means of a new cloud-based platform with a 2-way communication system (Sharesource), offering accurate daily monitoring of the therapy as well as ability to remotely alter the prescription. Its main documented advantage is the early identification of clinically relevant issues such as catheter dysfunction and nonadherence to prescribed PD therapy; its contribution to recognition of an imminent peritonitis has still to be evaluated. RPM is also of particular interest for patients with social, geographical or physical limitations in terms of travel reduction. Moreover, permanent access to data for PD teams may provide reassurance and alleviate anxiety generated by the remote nature of the method for both patients and nephrologists. Finally, RPM offers further advantages such as a more personalized APD prescription, tailored to patients' needs, as well as improved clinical follow-up and a more proactive care. Long-term impacts of RPM in PD on patients' outcomes, healthcare costs, and its potential influence on a greater take-up of the technique, notably through reinforced confidence for both patients and nephrologists through better visibility of the progress of the therapy, have still to be evaluated. Given the positive impact of PD on the preservation of residual renal function and association of the latter with better survival, any tool that may contribute to its broader use is most valuable.
\end{abstract}


Telemedicine and remote patient management (RPM) have been of growing interest in the last decades, and have been implemented with a significant positive impact in the care of chronic patients in many medical fields, including cardiology, diabetology, neurology, and pneumology [1]. In nephrology, home-based dialysis therapies, offering patients more flexibility and empowerment in comparison to in-center hemodialysis, have increasingly been developed. Peritoneal dialysis (PD) has been recognized as a highly valuable method of renal replacement therapy (RRT) as it provides, in comparison to hemodialysis, a better preservation of residual renal function [2], which is associated in the literature with better survival [3]. However, global acceptance and use of PD remains low with only $11 \%$ of incident end-stage renal disease (ESRD) patients, compared to $85 \%$ for hemodialysis (the remaining $4 \%$ being pre-emptively transplanted), according to recent data [4]. Although reasons have not been clearly identified yet, clinicians' lack of confidence about PD use because of problematic visibility to patient- and therapy-related issues, such as adherence to treatment and catheter dysfunction potentially hindering the smooth progress of therapy, as well as patients' fear to be left alone at home are plausible explanations. In automated peritoneal dialysis (APD), an RPM cloud-based platform with a 2-way communication system such as Sharesource, offering accurate monitoring of the therapy and ability to remotely alter the prescription, could potentially help to overcome some of these reluctancies.

In Switzerland, RPM in APD, by means of the SHARESOURCE platform, was implemented for the first time in December 2015 in the care of a 23-year-old ESRD patient who required hospitalization for urgent start of RRT because of symptomatic uremia [5]. She was known for prior non-adherence to treatment and regularly missed medical visits. Upon her firm wish to do PD, a catheter was rapidly inserted and low-volumes PD started without complications. Upon returning home, her therapy was remotely observed, thanks to the platform's interactive interface, which provides a dashboard displaying the exhaustive list of the PD clinic's patients as well as flags symbolizing for each of them the progress of the daily performed therapy based on predefined alarm parameters (green when uneventful, yellow or red in case of minor or major issues, respectively). Analysis of the daily-transmitted data in her case enabled early identification of 2 clinically relevant occurring issues.

Initially, the course of the PD therapy was unremarkable (as indicated by green symbols), but then red flag alerts appeared on the dashboard, attesting the occurrence of a major issue. Analysis of the cycle volume profiles showed recurrent prolonged drainage times. The patient was contacted for urgent clinical evaluation and radiological investigations. Catheter migration was quickly diagnosed and required laparoscopic repositioning after an unsuccessful attempt of 
conservative treatment using laxatives. Afterwards, red flags disappeared from the dashboard and cycle volumes profiles were unremarkable, indicating normal progress of PD therapy. Because catheter dysfunction represents a major cause of PD drop-out and permanent transfer to hemodialysis in the first 3-6 months of therapy [6,7], its identification is of utmost importance; any tool potentially contributing to an earlier diagnosis is of value for clinicians.

Sharesource also provides monthly overview graphs giving a general impression about the course of PD therapy, which, in the case of the aforementioned patient, was uneventful after the surgical correction of catheter migration. However, a few months later, empty boxes (i.e., devoid of symbols) appeared in the monthly overview graph, documenting the occurrence of non-adherence, which is defined as the performance of less than $90 \%$ of prescribed therapy; its early recognition enabled the PD team to quickly address this important issue. Indeed, non-adherence has been proved to have a major negative impact on clinical outcomes, as it is strongly associated with technique failure, rate and length of hospitalization as well as peritonitis and death [8-10]. According to recent data, its prevalence is high and extremely variable in PD patients, ranging from 2.6 to $53 \%$ for dialysis procedures [11]; young patients seem to be particularly at risk [12], possibly because they are more socially and professionally active and therefore confronted with more logistic challenges. In this case, the Sharesource monthly graph was shared with the 23-year-old patient to start an open discussion about this sensitive subject. Because it was presented as a positive tool to improve her care instead of the reflection of her poor adherence, she was not offended and did not feel her privacy was disturbed by RPM. On the contrary, it helped her to realize that missed therapies happened more frequently than she thought. After thorough explanations about the aforementioned potential shortand long-term issues, non-adherence to PD therapy resolved and monthly overviews were unremarkable in the following months.

Among PD complications, peritonitis remains one of the most frequent and most feared by nephrologists, due to its major negative impact on technique survival and transfer to permanent hemodialysis [13], as well as being a described risk factor for the occurrence of the rare but potentially life-threatening encapsulating peritoneal sclerosis through modifications of the characteristics of the peritoneal membrane [14]. Based on the postulate that ultrafiltration (UF) decreases during an acute episode $[15,16]$, a Mexican study retrospectively analyzed data in 10 patients and found a significant drop in UF $24 \mathrm{~h}$ before the occurrence of symptoms and a subsequent diagnosis of peritonitis [17]. It could, therefore, be a hint to an imminent episode, although the practical implication of this observation (e.g., by setting alarm parameters) may be difficult partly due to inter- and intra-individual variability of daily UF. 
Apart from early detection of clinically relevant issues, RPM in APD offers further practical advantages. Indeed, as it allows for both remote observation and alteration of therapy, the need for physically coming into the PD unit is markedly reduced, which is of particular interest, not only for patients with social, geographical or physical limitations raising organizational difficulties, but also for those desiring to spend their time more efficiently; however, because clinical evaluation and communication with the patient remain the keystones of good care, a regular in-person visit, classically on a monthly basis, should be maintained.

Although home-based treatment such as PD is a particularly attractive concept, notably for active patients who desire more flexibility and control or for populations with reduced mobility or hampered by other limitations, the remote nature of at-home dialysis and the fear of being left alone may generate anxiety and thus hold them back from adopting it. A RPM platform such as Sharesource, through its permanent access for PD teams to data about the performed therapy, may provide patients reassurance and more confidence in embracing this method by attenuating this feeling of being left in a "black hole" in comparison with standard in-center hemodialysis. This may also be true for clinicians, whose worries about potentially being unaware of issues occurring at home could represent an obstacle to the implementation of $\mathrm{PD}$ in the care of their ESRD patients.

Clinical follow-up can also be further improved by APD cycler-embedded RPM as it can provide daily important clinical data such as blood pressure and weight values, which can be entered before the PD therapy is initiated. It proved to be of great help for a patient who presented to the PD unit with significant weight gain over a period of a week, and was clinically fluid overloaded with leg edema up to the knees and pathologic pulmonary auscultation. The PD prescription was altered to optimize UF, and the patient was then asked to document his daily weight. In the following days, the PD team could verify that the weight went progressively back to its dry value and remained stable thereafter.

Because of a tendency for recurrent fluid overload over the months that followed, this patient's PD therapy had to be regularly altered; remote prescription greatly facilitated patient management by sparing a lot of travel time. Moreover, the PD team did not hesitate to make minor changes to further optimize therapy, thereby leading to a real patient-tailored prescription.

An Italian retrospective case-control study recently examined the clinical utility of the implementation of RPM with the SHARESOURCE platform in the care of their APD patients [18]. They observed 37 RPM-APD patients, 16 incidents, and 21 prevalents switched from traditional APD (cyclers functioning with a card containing data of performed therapy and registered prescriptions). 
They documented the number of modifications in APD prescription over a period of 6 months before and after the switch, and separately analyzed incident and prevalent patients. They demonstrated significantly more changes in prescriptions in both incident and prevalent RPM-APD patients as well as less visits to the PD unit for the incident group. Moreover, over 50\% of major modifications, defined as having clinical relevance in terms of depuration or UF (minor changes aimed at reducing alarms) were done proactively in the incident group ( $42 \%$ in the prevalent group), that is, before a patient calling or presenting to the clinic. In summary, the major advantages of RPM documented in this study are: a more proactive type of care as well as a more personalized therapy, and a potential reduction in costs, at least for incident patients.

In summary, $\mathrm{PD}$, and especially APD, is a medical field in which the broader use of RPM may be of particular benefit. The newly available cloud-based platform, Sharesource, has been implemented in recent years in the care of many patients throughout Europe, initially in England and Switzerland then in Italy and most recently in France. Through accurate monitoring of therapy and remote altering of prescription, RPM allows PD teams to work in a more proactive manner as compared with standard APD as well as to prioritize patients with red flags alerts who may potentially need quick intervention. The major clinical advantage that has been documented until now is the early identification of relevant occurring issues, such as catheter migration and non-adherence. Whether this will play a role in the recognition of imminent peritonitis through a drop of UF before the occurrence of symptoms, or its practical feasibility have still to be investigated, but the concept is indeed interesting.

A further advantage that may have an impact on the quality of life is alleviating the burden of travel times, which is of particular importance for specific populations such as patients with reduced mobility or hampered by other limitations, but also for very professionally or socially active patients who wish to spend their time more efficiently. Reassurance and reinforced confidence in APD through accurate daily monitoring is also a key feature of RPM for both patients and nephrologists. Further improvement of clinical follow-up through availability of relevant data such as blood pressure and weight is also valuable. The main limitation currently, is potential voluntary or involuntary human errors, as the values have to be manually registered. Bluetooth enabling would be a considerable enhancement for the reliability of data and reduction of patient tasks.

As illustrated by the Italian study, RPM has the potential to offer a more personalized therapy, tailored to patients' needs. It may also have an impact on healthcare costs, owing to the reduction of the number of documented in-person visits in the incident RPM group. 
These advantages make RPM a very promising tool to help clinicians further improve the quality of care of APD patients. It should, however, not be used to represent a burden and a disturbance in their privacy; of note, no such complaint has been documented yet and patients seem to work well with their RPM. Furthermore, it remains a tool in the therapeutic armamentarium and should not under any circumstances become a substitute for clinical evaluation as the latter is, together with doctor-to-patient communication, a cornerstone of good care.

In the future, the long-term impacts of RPM on important patient outcomes such as morbidity, mortality, hospitalization rate, and quality of life, as well as on healthcare costs and on technique survival will have to be determined.

From a more global point of view, it will be interesting to see whether RPM may have an impact on global uptake of PD; indeed, given its significant positive impact on the preservation of residual renal function, and the latter being associated with better survival, any means that can reinforce confidence in embracing this technique and lead to its broader use is valuable.

\section{Acknowledgments}

I am grateful to Mrs. Teresa Gmür for her help in copyediting the manuscript.

\section{Disclosure Statement}

V.J.D. has received grant support and honoraria from Baxter Healthcare.

\section{References}

1 Steventon A, Bardsley M, Billings J, Dixon J, Doll H, Hirani S, Cartwright M, Rixon L, Knapp M, Henderson C, Rogers A, Fitzpatrick R, Hendy J, Newman S; Whole System Demonstrator Evaluation Team: Effect of telehealth on use of secondary care and mortality: findings from the whole system demonstrator cluster randomised trial. BMJ 2012;344:e3874.

2 Jansen MA, Hart AA, Korevaar JC, Dekker FW, Boeschoten EW, Krediet RT; NECOSAD Study Group: Predictors of the rate of decline of residual renal function in incident dialysis patients. Kidney Int 2002;62:1046-1053.

3 Bargman JM, Thorpe KE, Churchill DN: Relative contribution of residual renal function and peritoneal clearance to adequacy of dialysis: a reanalysis of the CANUSA study. J Am Soc Nephrol 2001;12:2158-2162.
4 Kramer A, et al: The European renal association - European dialysis and transplant association (ERA-EDTA) registry annual report 2015: a summary. Clin Kidney J 2018;11:108-122.

5 Drepper VJ, Martin PY, Chopard CS, Sloand JA: Remote patient management in automated peritoneal dialysis: a promising new tool. Perit Dial Int 2018;38:76-78.

6 Kolesnyk I, Dekker FW, Boeschoten EW, Krediet RT: Time-dependent reasons for peritoneal dialysis technique failure and mortality. Perit Dial Int 2010;30:170-177.

7 Béchade C, Guittet L, Evans D, Verger C, Ryckelynck JP, Lobbedez T: Early failure in patients starting peritoneal dialysis: a competing risks approach. Nephrol Dial Transplant 2014;29: 2127-2135 
8 Bernardini J, Nagy M, Piraino B: Pattern of noncompliance with dialysis exchanges in peritoneal dialysis patients. Am J Kidney Dis 2000;35:11041110.

9 Bernardini J, Piraino B: Compliance in CAPD and CCPD patients as measured by supply inventories during home visits. Am J Kidney Dis 1998;31: 101-107.

10 Mawar S, Gupta S, Mahajan S: Non-compliance to the continuous ambulatory peritoneal dialysis procedure increases the risk of peritonitis. Int Urol Nephrol 2012;44:1243-1249.

11 Griva K, Lai AY, Lim HA, Yu Z, Foo MW, Newman SP: Non-adherence in patients on peritoneal dialysis: a systematic review. PLoS One 2014; 9:e89001.

12 Yu ZL, Lee VY, Kang AW, Chan S, Foo M, Chan CM, Griva K: Rates of intentional and unintentional nonadherence to peritoneal dialysis regimes and associated factors. PLoS One 2016; 11:e0149784.

13 Perl J, Davies SJ, Lambie M, Pisoni RL, McCullough K, Johnson DW, Sloand JA, Prichard S, Kawanishi H, Tentori F, Robinson BM: The peritoneal dialysis outcomes and practice patterns study (PDOPPS): unifying efforts to inform practice and improve global outcomes in peritoneal dialysis. Perit Dial Int 2016;36:297-307.
14 Lee HY, Kim BS, Choi HY, Park HC, Kang SW, Choi KH, Ha SK, Han DS: Sclerosing encapsulating peritonitis as a complication of long-term continuous ambulatory peritoneal dialysis in Korea. Nephrology (Carlton) 2003;8(suppl):S33S39.

15 Krediet RT, Zuyderhoudt FM, Boeschoten EW, Arisz L: Alterations in the peritoneal transport of water and solutes during peritonitis in continuous ambulatory peritoneal dialysis patients. Eur J Clin Invest 1987;17:43-52.

16 Albrektsen GE, Wideroe TE, Nilsen TI, Romundstad P, Radtke M, Hallan S, Aasarod K, Oien C, Laegreid IK: Transperitoneal water transport before, during, and after episodes with infectious peritonitis in patients treated with CAPD. Am J Kidney Dis 2004;43:485-491.

17 Rojas M, Paniagua R, Ramos A: Help of Remote Patient Monitoring in the Assessment of Changes in Ultrafitration before, During and after a Peritonitis Episode in Patients on Automated Peritoneal Dialysis, ASN 2017. https://www.asn-online. org/education/kidneyweek/2017/program-abstract.aspx? controlId=2784136.

18 Milan Manani S, Crepaldi C, Giuliani A, Virzi GM, Garzotto F, Riello C, de Cal M, Rosner MH, Ronco C: Remote monitoring of automated peritoneal dialysis improves personalization of dialytic prescription and patient's independence. Blood Purif 2018;46:111-117.
Valérie Jotterand Drepper

Division of Nephrology, Geneva University Hospital

Rue Gabrielle-Perret-Gentil 4

CH-1205 Geneva (Switzerland)

E-Mail Valerie.JotterandDrepper@hcuge.ch 